\title{
Location of vital structures and available bone for the placement of dental implants in clinically edentulous patients: A cohort study
}

\author{
Aruna Wimalarathna', Indika Thilakumara', Vajira Jayasinghe', Prasad Amaratunga ${ }^{1}$, \\ Ruwan Jayasinghe ${ }^{3}$
}

${ }^{1}$ Department of Prosthetic Dentistry, Faculty of Dental Sciences, University of Peradeniya, ${ }^{2}$ Department of Oral Pathology, Faculty of Dental Sciences. University of Peradeniya, ${ }^{3}$ Department of Oral Medicine \& Radiology, Faculty of Dental Sciences, University of Peradeniya, Peradeniya, Sri Lanka

Purpose: The purpose of this study was to evaluate a cohort of edentulous Sri Lankan patients and to consider important anatomical landmarks and available bone seen in dental panoramic tomography (DPT) for the placement of implants.

Materials and Methods: A total of 152 clinically edentulous arches were selected from the DPTs of 81 patients. DPTs were taken following standard protocols. Radiographs were evaluated by a specialist for the following: the relative thickness of the soft tissue coverage of the alveolar ridge, location of the mandibular canal, mental foramen and the maxillary sinus, bone height at the maxillary canine, first premolar region, mandibular canine, and symphyseal region, and for the presence of buried roots or impacted teeth and foreign bodies.

Results: The mean age of the patients in the sample was $68.4 \pm 9.8$ years. The sample comprised $59.0 \%$ females and $41.0 \%$ males. Out of 152 edentulous arches, $75(49.0 \%)$ were mandibular and $77(51.0 \%)$ were maxillary arches. The mean soft tissue thickness was $2.2 \pm 0.89 \mathrm{~mm}$. The average bone height from the lower alveolar crest to the mandibular canal was $10.5 \mathrm{~mm}$ in the first molar region. The position of the mental foramen was at $21.1 \pm 5.32 \mathrm{~mm}$ on the right side and $22.4 \pm 4.63 \mathrm{~mm}$ on the left from the midline. The bone height from the floor of the maxillary sinus and the alveolar crest was $<5 \mathrm{~mm}$ in $73.3 \%$ of patients on the right side and $72.0 \%$ on the left side.

Conclusions: Standard conventional implants were possible in the anterior segment and only short implants were possible in the posterior segment for the majority of edentulous Sri Lankan patients without the risk of damaging vital structures and with no additional pre-implant corrective surgical procedures. The average readings of anatomical parameters revealed in this study will be useful in the clinical and radiographical assessment of edentulous patients for dental implants. (JOURNAL OF DENTAL IMPLANT RESEARCH 2021;40(4): 151-157)

Key Words: Radiographs, Panoramic, Edentulous, Implant therapy

\section{INTRODUCTION}

In the last 30 years, dental implant-based treatments have become a valuable treatment option for completely edentulous patients as an alternative to conventional den- tures ${ }^{1)}$. As such, dental implants are considered an important element in prosthodontic treatment planning and clinical management of edentulous patients. Therefore, the radiological assessment of patients awaiting implant therapy would be of an important tool in the pre-surgical

Received September 20, 2021, Revised December 22, 2021, Accepted December 23, 2021

(c) Journal of Dental Implant Research.

This is an open access article distributed under the terms of the Creative Commons Attribution Non-Commercial License

(http://creativecommons.org/licenses/by-nc/4.0) which permits unrestricted non-commercial use, distribution, and reproduction in any medium, provided the original work is properly cited.

Correspondence to: Aruna Wimalarathna, https://orcid.org/0000-0001-5265-3589

Department of Prosthetic Dentistry, Faculty of Dental Sciences, University of Peradeniya, Peradeniya, Kandy, Sri Lanka. Tel: +94812387504, Fax: +94812388948, E-mail: aaakwimalarathna@gmail.com 
assessment of patients. The implant treatment plan would be greatly influenced by the proximity of vital structures such as the mandibular canal, mental foramen, and maxillary sinus to the proposed implant site. The radiographic examination would reveal the relationship of such vital structures to the implant site. Further, the radiographic examination would also be useful in finding other relevant information such as the thickness of the soft tissue covering, presence of buried roots and/or impacted teeth, bone pathologies, and foreign bodies in relation to the proposed implant site ${ }^{2)}$.

DPT is a cost-effective and relatively "low-dose" radiological technique used in routine dental practice ${ }^{3)}$. This technique allows assessing the maxillary and mandibular arches and their supporting structures on a single image $^{4)}$. Therefore, DPTs are considered an imaging modality that is readily available, time-saving, having a broader anatomic coverage and a high patient acceptability ${ }^{5}$. Further, DPT provides the clinician with better information about the sinus floor position and the quality of bone in edentulous regions awaiting implant placement $t^{5}$.

The purpose of this study was to evaluate the suitability of clinically edentulous patients for implant therapy by assessing their preliminary DPT radiological records in relation to the location of vital structures and available bone in the oro-antral region. The findings of this study would be beneficial to select the type of implant, the number of implants, their locations, the sizes of the implants, and the type of the prosthesis.

\section{MATERIALS AND METHODS}

A total of 152 clinically edentulous arches of DPTs of 81 patients were selected for this study. Those radiographs had been taken for different purposes during the 5-year period from 2016 to 2020. Most of these patients had visited the Department of Prosthetic Dentistry, either for the fabrication of new removable dentures or for correction of problems associated with their existing dentures.

Poor quality radiographs and the DPTs has taken following surgical or medical interventions less than 6 months were excluded from the study. The DPTs from the patients with single or both complete edentulous arches were included in the study. Ethical clearance was obtained from the Ethics Review Committee of the Faculty of Dental Sciences (ERC No: FDS-FRC/2014/09).

All the digital DPTs had been taken by using Vatech Pax-Duo 3D Cone Beam and Panoramic X-Ray machine with standard radiographic exposures and were evaluated by a specialist with higher training in maxillofacial radiology. Tools in the radiological imaging software (Ezdent) were used to measure the distances without any magnification to find out the suitability of these patients for implant-supported fixed or removable prosthesis by taking the following radiographic findings into consideration: 1) the relative thickness of the soft tissue coverage of edentulous ridge, 2) the location of the mandibular canal in rotation to the first molar region, 3) the position of the mental foramen 4) available bone height in relation to the maxillary sinus, 5) presence of buried roots or impacted teeth and foreign bodies.

The findings and the measured parameters were marked on a pre-designed labeled diagram of the maxilla and the mandible (Fig. 1). The midline, mental foramen, maxillary tuberosity, zygomatic process and canine fossa were used as anatomical landmarks for describing the location of the pathological findings in edentulous arches when necessary. In particular, the extents of the canine, premolars, and first molar regions were considered to be up to $1.5 \mathrm{~cm}, 2 \sim 2.5 \mathrm{~cm}$, and $3 \sim 3.5 \mathrm{~cm}$ from the midline respectively ${ }^{6}$. Data were entered into an Excel sheet and analyzed by using SPSS version 21 computer software.

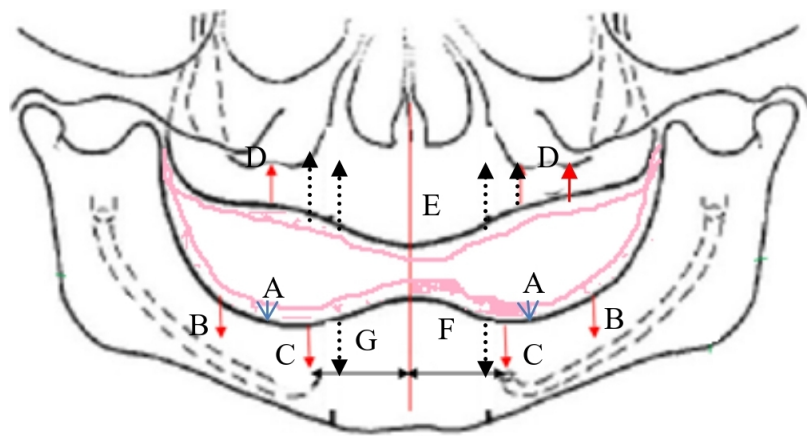

Fig. 1. Pre-designed labeled diagram of the maxilla and the mandibular area. 


\section{RESULTS}

The mean age of the study population was $68.4 \pm 9.8$ years with an age range of $45 \sim 96$ years. This sample consisted of $59.0 \%$ females and $41.0 \%$ males. Out of the 152 edentulous arches, 75 (49.0\%) were mandibular and 77 $\mathbf{( 5 1 . 0 \% )}$ were maxillary edentulous arches.

\section{Soft tissue thickness}

The mean soft tissue thickness of the first molar region when all four quadrants were taken together was $2.2 \pm 0.89$ $\mathrm{mm}$. The recorded maximum value of the average soft tissue thickness was $6.4 \mathrm{~mm}$. Out of all the cases the average soft tissue thickness was $<2 \mathrm{~mm}$ in $28.9 \%$ ( $\mathrm{n}=44$ ) cases, $2 \sim 4 \mathrm{~mm}$ in $67.8 \%(\mathrm{n}=103)$ cases, $4 \sim 6 \mathrm{~mm}$ in $2.6 \%$ $(\mathrm{n}=4)$ and $>6 \mathrm{~mm}$ in $0.7 \%(\mathrm{n}=1)$ in cases (Fig. 2).

\section{Mandibular canal}

The location of the mandibular canal was not clear in $36.0 \%$ on the right side, $38.0 \%$ on the left side, and $16.0 \%$

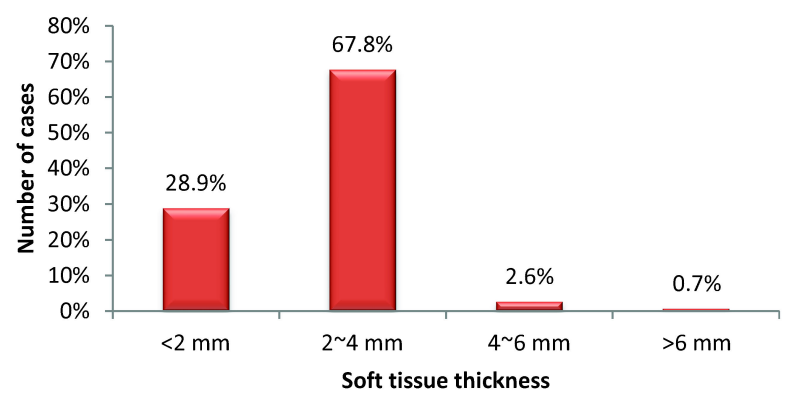

Fig. 2. Distribution of average gingival thickness.

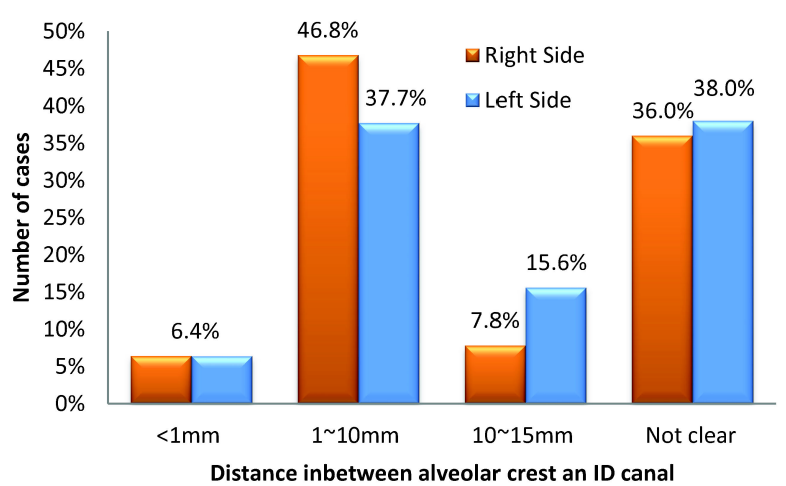

Fig. 3. The location of the inter-alveolar canal. on both sides. The distance from the crest of the alveolar bone to the inter-alveolar canal in both right and left sides concerning the first molar region was $<1 \mathrm{~mm}$ in $6.4 \%$ cases. That distance was 1 10 $\mathrm{mm}$ in $46.8 \%$ and $37.7 \%$ in right and left sides respectively. Among 7.8\% and $15.6 \%$ in the sample, the same parameter was between 10 15 $\mathrm{mm}$ in right and left sides respectively (Fig. 3).

\section{Mental foramen}

The horizontal distance from the midline to the mental foramen in right side was $21.1 \pm 5.32 \mathrm{~mm}$ and $22.4 \pm 4.82$ $\mathrm{mm}$ in left side. The vertical position of the mental foramen from the alveolar crest was $<5 \mathrm{~mm}$ in $16.0 \%(\mathrm{n}=12)$ in right and $10.6 \%(\mathrm{n}=8)$ in left side; $5 \sim 8 \mathrm{~mm}$ in $13.3 \%$ $(\mathrm{n}=10)$ similarly in both sides; that figure was in $8 \sim 10$ $\mathrm{mm}$ in $8.0 \%(\mathrm{n}=6)$ of right side and $14.6 \%(\mathrm{n}=11)$ in left side. That parameter was $>10 \mathrm{~mm}$ in $14.6 \%(\mathrm{n}=11)$ in right and $16.0 \%(n=12)$ in left side. The mental foramina

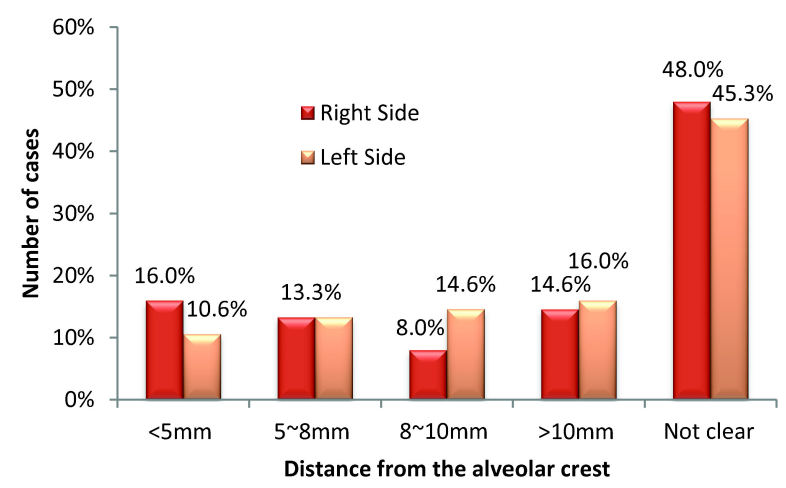

Fig. 4. Vertical position of the mental foramen.

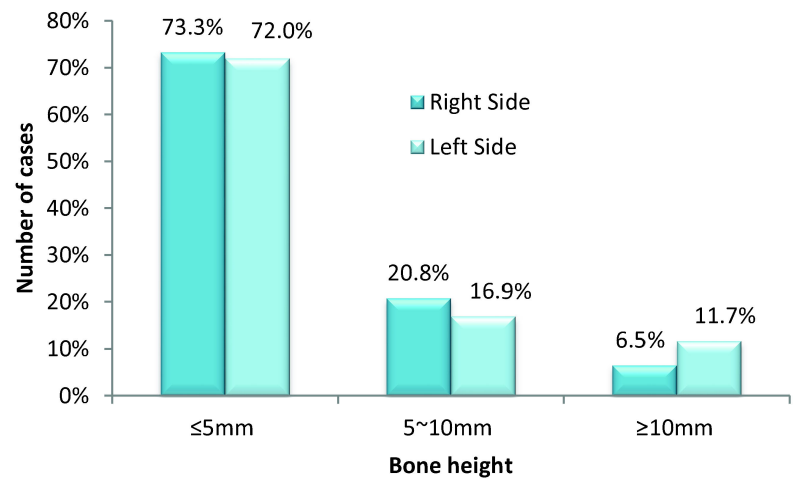

Fig. 5. The bone height in related to the maxillary sinus. 
were not clear in DPTs of $48.0 \%(n=36)$ and $45.3 \%(n=34)$ in right and left sides respectively (Fig. 4).

\section{Available bone height}

The bone height in relation to the maxillary sinus was $<5 \mathrm{~mm}$ in $73.3 \%(\mathrm{n}=56)$ in right side and $72.0 \% \quad(\mathrm{n}=55)$ in left side among reported cases in related to the first molar region. That parameter was $5 \sim 10 \mathrm{~mm}$ in $20.8 \%$ $(n=16)$ with $S D \pm 3.2$ in right side and $16.9 \%(n=13)$ with $\mathrm{SD} \pm 3.4$ in left side. The bone height is $>10 \mathrm{~mm}$ in $6.5 \%$ $(n=5)$ and $11.7 \%(n=9)$ in right and left side respectively (Fig. 5).

The bone heights in relation to the upper and lower canines, mandibular symphyseal, and upper first premolar regions are summarized in Table 1 . The average inter-canine distance is $26 \mathrm{~mm}$ in mandibular arches and $35 \mathrm{~mm}$ in maxillary arches.

Among the population, $9.7 \%, 7.2 \%$ and $0.7 \%$ were having completely buried roots, impacted teeth, and foreign bodies respectively.

\section{DISCUSSION}

Panoramic radiography is a widely used investigation tool in routine dental screening procedures. Especially in dental implant treatment planning, DPT could be considered the most convenient and widely available preliminary assessment tool. DPTs can be used to assess the status of the residual ridge, the position of the maxillary sinus, mandibular canal, and mental foramen as well as soft tissue thickness at the proposed implant site. Further, DPT would be very useful in finding out the presence of various pre-existing intra-bony pathological lesions, sep- tic foci, presence of unerupted teeth in relation to the proposed implant site.

There are two gingival biotypes. If the gingival thickness is $2 \mathrm{~mm}$ or more it is considered a thick biotype and if it is less than $1.5 \mathrm{~mm}$ is a scalloped/thin biotype. Therefore, the soft tissue thickness is directly related to the mucosal biotype. The mucosal biotype is an important factor affecting the implant success ${ }^{7,8)}$. Furthermore, it is the soft tissue thickness that determines the collar height of the gingival formers (healing abutments) of the implants during the healing phase ${ }^{9)}$.

According to findings of the current study, the overall average mucosal thickness at the crest of the alveolar ridge of the Sri Lankan edentulous patients was $2 \mathrm{~mm}$ (28.0\% cases) and $4 \mathrm{~mm}$ (68.0\% cases). A similar study on mucosal thickness of the same anatomical locations using ultrasonography has shown compatible results ${ }^{10,11)}$. This finding will be useful in determining the heights of gingival formers (healing abutments) that will be most required for Sri Lankan patients.

The Inferior Dental Nerve (IDN) is one of the most important vital structures in the lower arch. As such, the Inferior Alveolar Canal (IAC) is considered as an anatomical landmark of paramount importance in clinical implant dentistry when it comes to placement of implants in the posterior mandible. The position of the IAC is important in deciding the length and the angulation of the posterior mandibular implants. The distant in between the IAC and the crest of the alveolar bone determines the available bone height.

Results of the present study revealed that the available bone height in the posterior mandible is less than $10 \mathrm{~mm}$ in $47.0 \%$ of the patients in right side and $37.0 \%$ in the

Table 1. Bone heights in relation to canines, symphyseal and upper first premolar regions

\begin{tabular}{|c|c|c|c|c|c|c|c|}
\hline \multirow{3}{*}{ Variables } & \multicolumn{7}{|c|}{ Bone heights in predicted implant sites $(\mathrm{mm})$} \\
\hline & \multicolumn{3}{|c|}{ Mandibular $(n=75)$} & \multicolumn{4}{|c|}{ Maxillary $(n=77)$} \\
\hline & Right canine & Symphyseal & Left canine & $\begin{array}{l}\text { Right } 1^{\text {st }} \\
\text { premolar }\end{array}$ & Right canine & Left canine & $\begin{array}{l}\text { Left } 1^{\text {st }} \\
\text { premolar }\end{array}$ \\
\hline Mean & 17.4 & 19.2 & 17.2 & 10.6 & 14.7 & 15.0 & 9.8 \\
\hline Median & 18.5 & 19.6 & 18.6 & 10.2 & 15.0 & 14.7 & 9.5 \\
\hline SD & 5.17 & 4.96 & 5.52 & 3.27 & 3.62 & 3.63 & 3.69 \\
\hline$<10 \mathrm{~mm}$ & $9.3 \%(n=7)$ & $13.3 \%(n=10)$ & $13.3 \%(n=10)$ & $44.1 \% \quad(n=34)$ & $12.9 \%(n=10)$ & $12.9 \% \quad(n=10)$ & $46.7 \%(n=36)$ \\
\hline $11 \sim 20 \mathrm{~mm}$ & $58.6 \% \quad(n=44)$ & $44.0 \% \quad(n=33)$ & $57.3 \%(n=43)$ & $55.8 \%(n=43)$ & $84.4 \% \quad(n=65)$ & $81.8 \% \quad(n=63)$ & $53.2 \%(n=41)$ \\
\hline $21 \sim 30 \mathrm{~mm}$ & $32.0 \% \quad(n=24)$ & $42.6 \% \quad(n=32)$ & $29.3 \% \quad(n=22)$ & $0.0 \%(n=0)$ & $2.5 \%(n=2)$ & $5.1 \%(n=4)$ & $0.0 \% \quad(n=0)$ \\
\hline
\end{tabular}


left sides. Therefore, clinicians will have to settle for implants of less than $10 \mathrm{~mm}$ in managing posterior mandible. Implants less than $10 \mathrm{~mm}$ in length are considered as short implants ${ }^{12}$ with lesser bone implant contact. Therefore, when short implants are warranted, it is important to consider wider ( $>5 \mathrm{~mm}$ in diameter) implants whenever possible, in order to obtain greater bone implant contact and thereby to achieve a better initial stability. Furthermore, "All-On-Four" treatment option should be considered for patients with severely compromised posterior ridge. The "All-On-Four" treatment concept was developed to maximize the use of available remnant bone in atrophic jaws. The protocol uses four implants in the anterior part of complete edentulous jaws to support either a provisional, fixed or immediate loading prosthesis ${ }^{13)}$.

Results of the present study revealed that only a small number $(7.8 \%$ and $15.6 \%$ in right and left sides respectively) of patients were suitable for long implants and a majority $(>50 \%)$ of Sri Lankan edentulous patients were suitable only for short implants. The similar findings were revealed in Nemati and colleagues study also $^{14)}$.

The location of the mental foramina has been used to divide the mandible into 3 segments namely, the anterior segment and the left and right posterior segments. According to the Misch-Judy classification, the right and left posterior segments extend from the mental foramen to the retromolar pad and the anterior segment lies between the mental foramina ${ }^{15}$. Further, the available bone in the anterior mandible has also been divided into five equal columns of bone vertically to denote potential implant sites ${ }^{16)}$. Therefore, the location of mental foramina is an important finding in pre-surgical assessment of the mandible in deciding the number, size and angulations of implants and the type of implant-supported overdentures.

In the current study, mental foramina were not clear in $45.0 \%$ of DPTs making it difficult to identify the 3 segments of the mandible and to decide what type of the overdenture according to Mish classification. But most subjects in the study sample were having substantial bone height in the anterior regions making them suitable candidates for the long implant-supported conventional or cantilevered overdentures.

The position of the maxillary sinus and available alveolar bone below the sinus floor is the key determinant in planning implant treatment in the maxillary posterior segments. Due to pneumatization following extractions, the size and the shape of the maxillary sinus can vary considerably in edentulous maxilla. Accordingly, the amount of available bone in between the sinus floor and the alveolar crest for placement of implants vary from patient to patient and between different sites of the posterior alveolar segment of the same patient. Depending on the bone height between the floor of the sinus and the alveolar crest in the posterior maxilla, patients can be placed into 3 categories; the available bone height (1) more than $10 \mathrm{~mm}$, (2) between 6 to $9 \mathrm{~mm}$. (3) less than $5 \mathrm{~mm}$. The implant treatment protocol varies depending on the available bone height. If more than $10 \mathrm{~mm}$ of bone is available, then a suitably sized implant can be placed without sinus augmentation. If the bone is inadequate there are two different techniques of sinus lift to enhance the bone volume; direct and indirect ${ }^{13)}$. If the bone height is between 6 to $9 \mathrm{~mm}$, then indirect sinus augmentation and the placement of implant can be done simultaneously. However, if the available bone height below the sinus floor is less than $5 \mathrm{~mm}$, a direct sinus lift or a 2-stage indirect sinus lift should be performed 8 months prior to the placement of the implant.

However, independent implants and cantilever bars are usually not an option during implant-supported overdentures in edentulous maxilla due to less bone density and severely compromised force directions ${ }^{15}$. Therefore, only two varieties of overdentures are limited to edentulous maxilla. These are either, hybrid overdentures with $4 \sim 6$ anterior implants and posterior soft tissue supported overdentures or fully implant borne overdentures with 7 $\sim 10$ anterior and posterior implants. According to the current study, around $82.0 \%$ of individuals have $11 \sim 20$ $\mathrm{mm}$ bone height in relation to their canine regions and more than $53.0 \%$ them have the same amount of bone height in their upper first premolar regions (Table 1). Therefore, when considering the bone availability of this study sample, most of them were suitable candidates for the first option while $10.0 \%$ of the population indicated for the second option without sinus augmentation. 
The other extremity of the overdentures for the edentulous mandible is single implant-retained overdenture. Usually, the single implant is placed in the mandibular symphyseal region. In the study done by Mahoorkar et al, the majority of the studies supported the concept of single implant overdentures ${ }^{16)}$. Further, Cheng et al, has shown significant improvement of all parameters of patient satisfaction and chewing efficiency $(\mathrm{P}<0.001$ to 0.043) after attachment of the mandibular single implant retained overdentures ${ }^{17)}$. According to the present study, $90.0 \%$ of individuals have $>11 \mathrm{~mm}$ bone height in the mandibular symphyseal region (Table 1). Therefore, considering the difficult financial circumstances of patients in developing countries it seems to be a useful overdenture option for the management of edentulous lower jaws.

\section{LMITATIONS}

The edentulousness period of the participants were not considered during the selection for the study.

\section{CONCLUSION}

Standard conventional implants were possible in the anterior segment and only short implants were possible in the posterior segment of majority of edentulous Sri Lankan patients without a risk of damaging vital structures and with no additional pre implant corrective surgical procedures. Average readings of anatomical parameters revealed in the present study will be useful in clinical and radiographical assessment of edentulous patients for dental implants.

\section{AVAILABILTYY OF DATA}

All data generated or analyzed during this study are included in this published article.

\section{CONFLICT OF INTERESTS}

Herewith all the authors declared that there is no any conflict of interests.

\section{ORCID}

Aruna Wimalarathna,

https:/ / orcid.org/0000-0001-5265-3589

Indika Thilakumara,

https:/ / orcid.org/0000-0002-0525-6801

Vajira Jayasinghe,

https:/ / orcid.org/0000-0002-0660-1910

Prasad Amaratunga,

https:/ / orcid.org/0000-0002-5771-1212

Ruwan Jayasinghe,

https:/ / orcid.org/0000-0002-8054-4301

\section{REFERENCES}

1. Mañes Ferrer JF, Fernández-Estevan L, Selva-Otaolaurruchi E, Labaig-Rueda C, Solá-Ruíz MF, Agustín-Panadero R. Maxillary Implant-Supported Overdentures: Mechanical Behavior Comparing Individual Axial and Bar Retention Systems. A Cohort Study of Edentulous Patients. Medicina (Kaunas) 2020;56:139.

2. Jindal SK, Sheikh S, Kulkarni S, Singla A. Significance of pre-treatment panoramic radiographic assessment of edentulous patients-A Survey. Med Oral Patol Oral Cir Bucal 2011;16:e600-6.

3. Choi JW. Assessment of panoramic radiography as a national oral examination tool: review of the literature. Imaging Sci Dent 2011;41:1-6.

4. Awad EA, Al-Dharrab A. Panoramic radiographic examination: a survey of 271 edentulous patients. Int J Prosthodont 2011;24:55-7.

5. Kose TE, Demirtas N, Cakir Karabas H, Ozcan I. Evaluation of dental panoramic radiographic findings in edentulous jaws: $\mathrm{A}$ retrospective study of 743 patients "Radiographic features in edentulous jaws". J Adv Prosthodont. 2015;7:380-5.

6. Hashim HA, Al-Ghamdi SAF. Tooth Width and Arch Dimensions in Normal and Malocclusion Samples: An Odontometric Study. J Contemp Dent Pract 2005;6:36-51.

7. Thoma DS, Buranawat B, Hämmerle $\mathrm{CH}$, Held U, Jung RE. Efficacy of soft tissue augmentation around dental implants and in partially edentulous areas: a systematic review. J Clin Periodontol 2014;41:S77-91.

8. Chen ST, Buser D. Clinical and esthetic outcomes of implants placed in postextraction sites. Int J Oral Maxillofac Implants 2009;24:186-217.

9. Ferrari M, Cagidiaco MC, Garcia-Godoy F, Goracci C, Cairo F. Effect of different prosthetic abutments on peri-implant soft tissue. A randomized controlled clinical trial. Am J Dent 2015;28:85-9.

10. Dong J, Zhang FY, Wu GH, Zhang W, Yin J. Measurement of mucosal thickness in denture-bearing area of edentulous mandible. Chin Med J (Engl) 2015;128:342-7. 
11. Dong J, Zhang FY, Wu GH, Zhang W, Yin J. Measurement of mucosal thickness in denture-bearing area of edentulous mandible. Chin Med J (Engl) 2015;128:342-7.

12. Annibali S, Cristalli MP, Aquila DD, Bignozzi I, Monaca GL, Pilloni A. Short Dental Implants: A Systematic Review. Journal of Dental Reasearch 2012;91:25-32.

13. Peñaloza DS, Alonso RZ, Diago MAP, Diago MP. The all-on-four treatment concept: Systematic review. J Clin Exp Dent 2017;9:e474-88.

14. Nemati S, Moghadam AA, Kajan ZD, Mohtavipour ST, Amouzad H. An Analysis of Visibility and Anatomic Variations of Mandibular Canal in Digital Panoramic Radiographs of
Dentulous and Edentulous Patients in Northern Iran Populations. J Dent (Shiraz) 2016;17:112-20.

15. Misch CM: Classification of partially and completely edentulous arches in implant dentistry. In Misch CE, editor: contemporary implant dentistry, St Louis, 1993, Mosby.

16. Mahoorkar S, Bhat S, Kant R. Single implant supported mandibular overdenture: A literature review. J Indian Prosthodont Soc 2016;16:75-82.

17. Cheng T, Li Ma, Liu XL, Sun GF, He XJ, Huo JY, Wang YN. Use of a single implant to retain mandibularoverdenture: A preliminary clinical trial of 13 cases. Journal of Dental Sciences 2012;7:261e266. 